\title{
Somatic healthcare utilisation patterns among older people with intellectual disability: an 11-year register study
}

\author{
Magnus Sandberg ${ }^{1 *}$ D, Gerd Ahlström ${ }^{1}$, Anna Axmon ${ }^{1,2}$ and Jimmie Kristensson ${ }^{1,3}$
}

\begin{abstract}
Background: People with intellectual disabilities (ID) are known to have more diseases and are believed to start aging earlier than the general population. The population of older people with ID is growing, but knowledge about their use of healthcare is limited. This study aimed to explore somatic healthcare utilisation patterns among people with ID living in Sweden, in comparison with the general population from 2002 to 2012.

Methods: Participants were a group of people with ID $(n=7936)$ aged 55 years and older in 2012 , and an equal-sized, birth year and sex matched, general population sample $(n=7936)$. Participants were divided into age groups of 5-year intervals. Data regarding in- and outpatient care were collected from the Swedish National Patient Register.

Results: In the younger age groups, the ID group had higher healthcare utilisation compared with the general population sample, with higher risks for planned and unplanned somatic in- and outpatient care, particularly for unplanned inpatient registrations. Decreasing patterns were seen with age; with lower risks in the ID group for the oldest age groups. This was most evident in planned somatic in- and outpatient care. In those with at least one registration, the ID group had a longer unplanned length of stay in the younger age groups, but fewer planned visits to physicians in somatic outpatient care compared with the control group.

Conclusions: Compared with the general population, people with ID show higher healthcare utilisation in younger age groups. Healthcare utilisation decreases with age, and in old age, fewer people with ID use healthcare compared with the general population. The barriers to accessing planned healthcare for older people with ID need more investigation.
\end{abstract}

Keywords: Aged, Aging, Healthcare utilisation, Inpatient, Intellectual disability, Outpatient, Register studies, Retrospective studies

\section{Background}

Knowledge about healthcare needs, healthcare utilisation and access to healthcare among older people with intellectual disabilities (ID) is sparse. The number of older people with ID is increasing rapidly [1] and they experience high rates of multi-morbidity [2,3]. Therefore, it is important to gain a deeper understanding about the healthcare needs of people aging with ID, and the health system's ability and readiness to meet these needs.

\footnotetext{
* Correspondence: Magnus.Sandberg@med.lu.se

${ }^{1}$ Department of Health Sciences, Faculty of Medicine, Lund University, P.O.

Box 157, SE-221 00 Lund, Sweden

Full list of author information is available at the end of the article
}

People with ID differ in terms of overall health compared with the general population, and have a higher prevalence of chronic conditions such as diabetes mellitus [4], cardiovascular diseases [5], osteoporosis [6], visual and hearing impairments $[7,8]$, epilepsy, musculoskeletal diseases [8], and thyroid conditions [7]. They also experience high rates of health risk factors such as obesity $[9,10]$, physical inactivity, sedentary lifestyles, and inadequate emotional support [4], and are reported to have high, or undetected, healthcare needs $[8,11-15]$. However, as only one of these previous studies [9] focused on older people, more research is needed on this topic. 
The aging process is believed to start earlier in people with ID, which causes earlier onset of age-related diseases $[7,13,16,17]$ and a corresponding increase in healthcare needs. The World Health Organization has expressed concern that the increasing proportion of older people in general means that limited healthcare resources will be primarily used to satisfy general population needs, and only secondarily for the needs of people with ID [18]. This is problematic, as people with ID are already disadvantaged in accessing healthcare and social services [18]. Therefore, studies that compare the utilisation of healthcare in older people with ID with that of the general population are important.

Various forms of healthcare utilisation in people with ID have been internationally investigated; for example, hospital admissions, use of social services, primary care, outpatient specialist care, and rehabilitation services $[15,19-4041,42]$. However, most of these studies have limitations in terms of determining casual relationships, such as being cross-sectional [15, 19-32, 34-38], focusing on only one type of utilisation $[15,20-23,25,27-32,35$, $37,41]$, small sample size [23, 24, 27, 33, 37], or they included people of younger ages [21, 22, 28-35, 40]. Therefore, the current evidence base is weak. To our knowledge, only one study focusing on healthcare utilisation across time in people aging with ID has been conducted [41].

Previous research has shown that $10-19 \%$ of people with ID are admitted to hospital each year [26, 30, 33, 34, $36,41,42]$. The average length of stay (LOS) is reported to be 3.7-6.7 days [19, 28, 33, 41], although one study found a mean LOS of 16.9 days [28]. However, only two of these studies were longitudinal $[33,41]$, and only three made comparisons with a general population sample or official general population statistics [19, 41, 42]. Studies investigating outpatient care have reported that $82-92 \%$ of those with ID had at least one outpatient/general practitioner visit in the past 12 months $[15,34]$ and had an average of 1.3-5.4 annual contacts [15, 23, 37].

In summary, there are few available studies investigating inpatient and outpatient healthcare utilisation among older people with ID that have a large sample, a longitudinal design, and a matched control group. Such studies are important to detect changes over time, make valid interpretations of healthcare utilisation patterns, and determine if these patterns differ between people with ID and the general population. Most previous studies also investigated one specific form of healthcare utilisation. However, as people with ID may have their health needs met in various parts of the health system, a more comprehensive investigation is needed that covers both inpatient and outpatient care.

Therefore, this study aimed to explore somatic healthcare utilisation patterns among people with ID living in
Sweden compared with the general population from 2002 to 2012.

\section{Methods \\ Study design}

This was a longitudinal, retrospective, population registerbased study that included people with ID and a birth yearand sex-matched general population sample.

\section{Setting}

The population in Sweden is about nine million, and about $18 \%$ of them are aged 65 years or older [43]. Sweden has also one of the ten highest average life expectancies in the world with an average life expectancy of almost 84 years for women and just below 80 years for men in 2011 [44].

Sweden has a welfare system mainly funded by taxes and thus, the Swedish population is covered by a national health insurance and by this, have equal access to healthcare [45]. Because of this private health insurances are less common in Sweden, with only four percent that have such an insurance. These are also mostly common within occupational healthcare services and are therefore in most cases paid for by employers [45]. Healthcare and social services are regulated by three laws: the Health and Medical Services Act [46], the Act concerning Support and Service for Persons with Certain Functional Impairments [47], and the Social Services Act [48]. Healthcare and social services are provided by municipalities and county councils at different levels. In Sweden there are 21 county councils that are responsible at a regional level for providing healthcare services to their populations (including specialized medical care in outpatient and inpatient facilities, rehabilitation and home nursing care) [45]. The 290 municipalities provide local-level healthcare and social services and is responsible to meet the care and housing needs of older people and people with disabilities. Care is provided in either the person's own home or in special accommodation/group homes. All healthcare and social services provided within these three laws by the county councils and muncipalities are recorded in mandatory public national registers.

Provision of inpatient and outpatient specialist care related to the Health and Medical Services Act [46] is registered in theSwedish National Board of Health and Welfare's National Patient Register (NPR). The purpose of this patient registry is to follow health trends in the population, improve the prevention and treatment of diseases, and contribute to the development of healthcare. The registry provides statistics from 1964 for the evaluation of healthcare and research.

Support and services provided under the Act concerning Support and Service for Persons with Certain Functional Impairments [47] are recorded in the National Board of 
Health and Welfare's LSS-register. One part of this register is a three-group classification, or personae, that classify the impairment of the service user. Persona 1 represents people who have ID from birth or an early age, with autism or conditions similar to autism; persona 2 represents people who have considerable and permanent mental impairment following brain damage as an adult; and persona 3 represents people who as a result of other serious and permanent functional disabilities, which are clearly not the result of normal ageing, have considerable difficulties in everyday life and great need of support or service [47].

\section{Study populations}

The ID group comprised all people who received support and social services according to the Act concerning Support and Service for Persons with Certain Functional Impairments [47] during 2012, which were registered as persona 1 , and who by the end of that year were aged 55 years or older. It is believed that the aging process is likely to start earlier in people with ID [18], but how much earlier is not known and most likely varies with type of ID. We chose a threshold of 55 as it seemed reasonable in relation to this and to the definition of old used for the general population, i.e., 65 years of age. The ID population was identified through the national LSS-register.

A randomly selected control group comprising people from the general population, matched by birth year and sex, was identified from the Swedish National Population Register [49]. We did not match on socioeconomic variables such as income level or educational level. These may be parts of the casual chain between ID and healthcare utilisation. The matching procedure was carried out by Statistics Sweden [50]. The ID group comprised 7936 individuals, and with the equally large control group the study in total included 15,872 participants. A person that had been included in the ID group could not also be selected as a control.

\section{Material}

Anonymized in- and outpatient data were collected from the NPR for the 11-year period from January 1, 2002, to December 31, 2012. This included information about inpatient care (e.g., hospital/clinic, date of registration, whether or not the admission was planned, and LOS) and outpatient care (e.g., hospital/clinic, date of physician visit and whether or not the visit was planned). An unplanned registration/visit means that it was not scheduled and could therefore be acute or sub-acute. Every stay at a ward resulted in a registration. This means that if an individual changed wards during the hospital stay there would be several registrations for the same in-hospital period. Healthcare utilisation for somatic care was identified based on the clinic to which the individual had visited or been admitted. All healthcare utilisation information was registered by staff at the different healthcare facilities at the time of the visit. NPR data used in the present study can be found in Table 1.

\section{Statistical analysis}

Age cohorts at 5-year intervals were created for each study year. Differences between the ID and control groups were investigated with respect to four different outcomes: planned/unplanned inpatient registrations/ outpatient physician visits. For each outcome, year, and age group, odds ratios (OR) with $95 \%$ confidence intervals for at least one registration/visit were estimated using logistic regression (presented in the Additional file 1). To visualize patterns, ORs based on age groups with at least 100 participants were plotted for each year and outcome. In addition, repeated-measure analyses were performed, with ORs for differences between the ID and control groups calculated and plotted for the whole study period, regardless of year. To investigate ORs and age patterns within the ID group, repeated-measures analyses were conducted, using the largest group (aged 55-59 years) as the reference. The regression analyses were not controlled for any variables, as the groups were already age- and sex matched, and as no other background variables were available. Differences in number of visits were assessed by a Mann-Whitney $U$ test, as the data were severely skewed. However, as most medians and quartiles were equal for those with at least one registration/visit, means and standard deviations (SDs) are presented rather than medians and quartiles. Here, age groups were merged into 10-year intervals to allow presentation of the large amount of data. Only comparisons with at least five individuals in each group are presented. Changes between age groups were investigated with Chi-square tests for trends for nominal data, and Jonckheere-Terpstra tests for skewed ratio data.

All analyses were performed with IBM SPSS statistics for Window, Version 23 (IBM Corp, Armonk, NY, USA).

Table 1 Description of used variables in the National Patient Register

\begin{tabular}{lll}
\hline Inpatient care & Outpatient care & Used for: \\
\hline Hospital/Clinic & Hospital/Clinic & Identifying somatic healthcare \\
Date of registration & Date of physician visit & Determining year of utilisation and the number of registrations/visits \\
Planned/unplanned registration & Planned/unplanned visit & Determining whether or not the registration/visit was planned \\
Length of stay & - & Length of stay for each registration \\
\hline
\end{tabular}




\section{Results}

\section{Patterns of somatic healthcare utilization}

For all types of healthcare utilization investigated (planned/ unplanned in- and outpatient care), a decrease in utilization in the ID group compared with the control group was found with increasing age (Fig. 1a-d, details presented in the Additional file 1). Those in the younger age groups in the ID group were more likely to utilise healthcare than their peers in the control group, while the opposite was seen in the older age groups. This was most evident in planned outpatient visits (Fig. 1c). For unplanned inpatient visits (Fig. 1b), the ID group had a higher proportion of individuals with at least one registration in most age groups, with an OR below 1 only in the oldest age group.

The same patterns are also seen in Fig. 2, where the ORs for each age group are presented independently of year (details presented in the Additional file 1). This pattern was obvious for planned inpatient registrations (Fig. 2a) and planned outpatient visits (Fig. 2c), with ORs above 1 in the younger age groups and below 1 in the older age groups. Unplanned inpatient registrations (Fig. 2b) showed a decreasing trend, but an OR below 1 only in the oldest age group.

When examining the age effect within the ID group, with the group aged 55-59 years as the reference group, Fig. 3 shows less clear patterns with increasing age (details presented in the Additional file 1). For planned inpatient registrations, there was an increase until the age 70-74 years, and thereafter a decline (in relation to the group aged 55-59 years) (Fig. 3a). For unplanned inpatient registrations, there was a clear pattern of increasing OR with age (Fig. 3b). For planned outpatient visits, there was a small increase until the age 70-74 years, followed by a decrease (Fig. 3c). The OR for unplanned outpatient visits also showed a small increase with age when considering all age groups. However, between the groups aged $55-59$ years and 80-84 years, the OR was relatively stable (Fig. 3d).

\section{Planned somatic inpatient care and planned LOS for those with at least one registration}

Among those with at least one planned inpatient registration, less visits were found in the ID group than in the control group for those aged 45-54 years (in 2002) and 55-64 years (in 2011) (Table 2). There were no linear trends for age in planned inpatient registrations. For planned LOS, the ID group had longer stays for those aged 45-54 years (in 2002), 55-64 years (in 2008), and 75-84 years (in 2010) (Table 2). Planned LOS increased with age in the ID group in 2005 and 2010, and in the control group in 2004, 2009, and 2011.

\section{Unplanned somatic inpatient care and unplanned LOS for those with at least one registration}

Among those with unplanned inpatient registrations, some statistically significant higher values were seen in

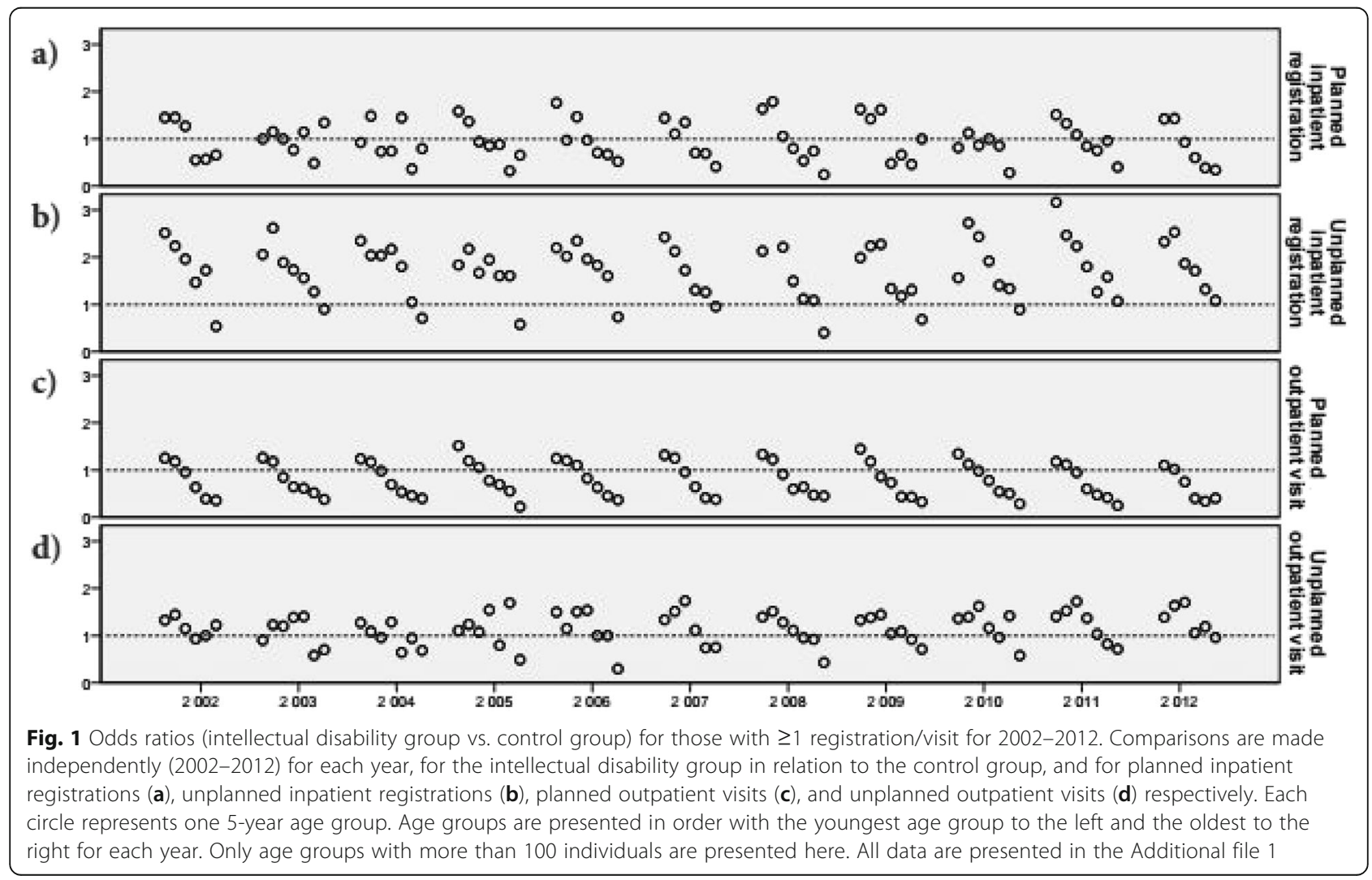




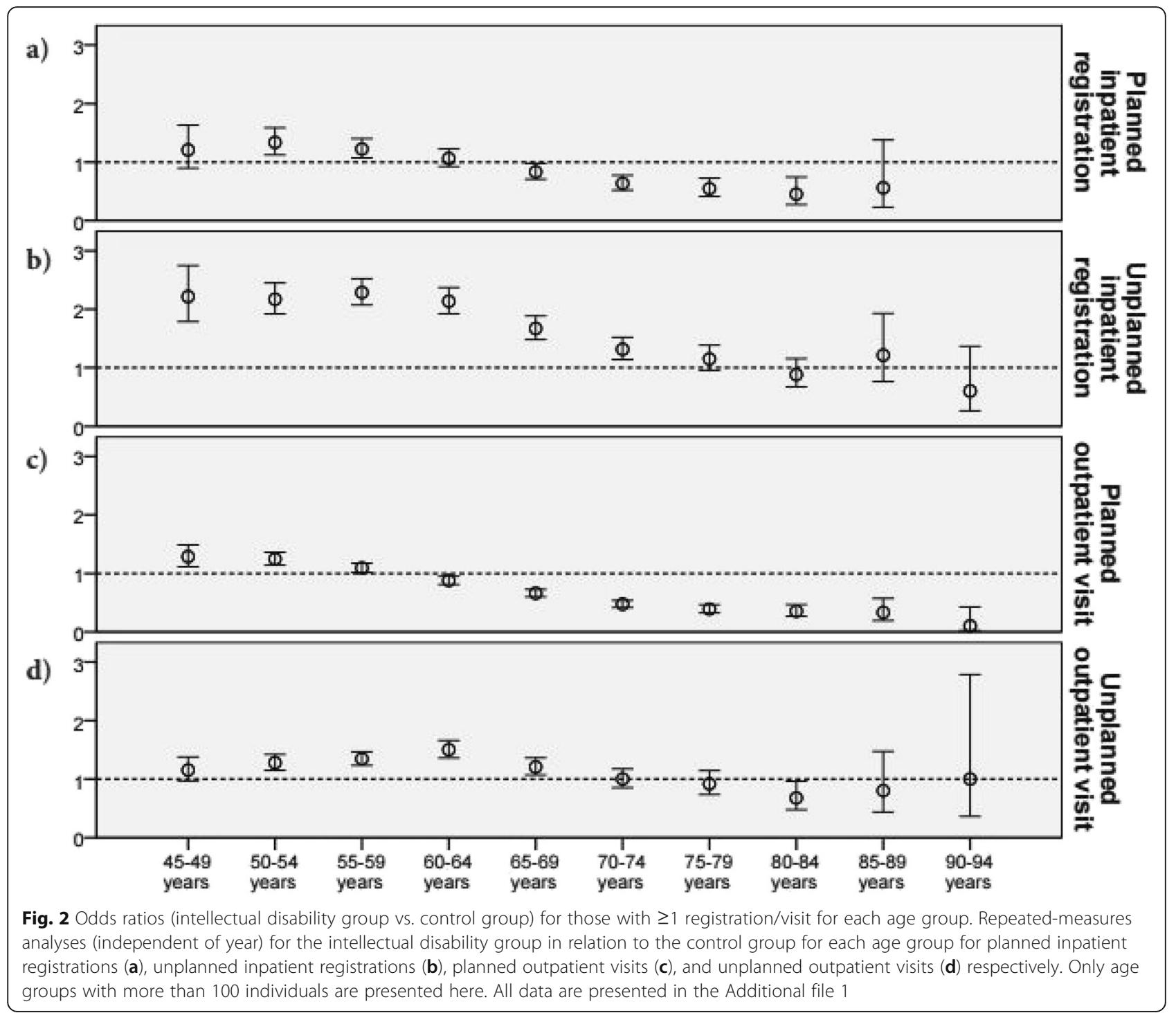

the ID group in the younger age groups (one in the group aged 45-54 years, four in the group aged 55-64 years, and one in the group aged 65-74 years) (Table 3 ). In the group aged $78-84$ years, one statistically significant difference was found, with higher values in the control group. Increasing trends were seen only in the control group for three of the study years $(2007,2011$, and 2012).

More statistically significant differences were seen when investigating unplanned inpatient LOS. For the three youngest age groups, the ID group had statistically significant higher means in 21 of 32 comparisons (stratified by year) (Table 3 ). No statistically significant differences were found in the two oldest age groups. There was a statistically significantly increasing trend for age in eight of the 11 study years in the ID group, and in seven of the 11 study years in the control group.
Planned physician visits in somatic outpatient care for those with at least one visit

When looking at the number of planned visits to physicians in outpatient care among those with at least one planned outpatient visit, there were 16 significant differences in total (four in the group aged 45-54 years, four in the group aged 55-64 years, six in the group aged 65-74 years, and two in the group aged 75-84 years); all of which showed lower values in the ID group compared with the control group (Table 4). There was an increasing trend with age in the control group in four of the 11 study years.

\section{Unplanned physician visits in somatic outpatient care for those with at least one visit}

For those with at least one unplanned visit to physician in outpatient care, statistically significant higher values were found in the ID group compared with the control 


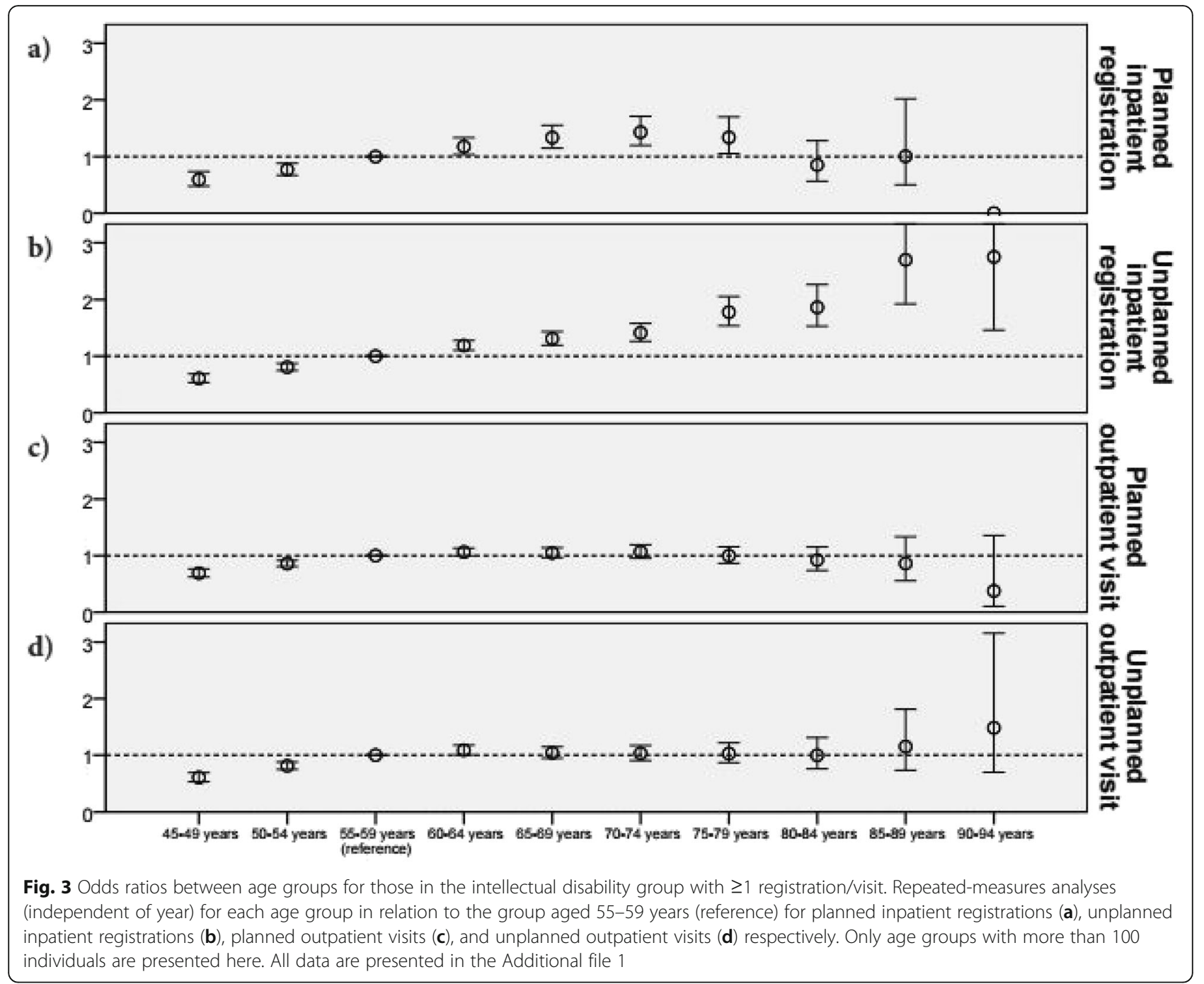

group in the youngest age group (45-54 years) in four of the 11 study years, the group aged 55-64 years in three of the study years, and for one of the study years each in the groups aged 65-74 years and 75-84 years (Table 4). For unplanned physician visits in outpatient care, there was only one statistically significant positive trend with age in the control group in 2009.

\section{Discussion}

The results of the present study showed that people with ID have a different healthcare utilisation pattern with a decreasing risk of healthcare utilisation with age compared with the general population.

In general, younger people with ID utilise more unplanned and planned healthcare in terms of inpatient registrations and outpatient care visits compared with the same age groups in the general population (Fig. 1). This is not surprising, as people with ID have higher rates of many diseases [3-8], many of which may cause planned or unplanned physician visits for outpatient care or hospital admissions. The more complex disease patterns in people with ID may also be a reason for the longer LOS in those with at least one inpatient registration than in the general population (Table 3).

In the oldest age groups, fewer people in the ID group utilised healthcare compared with the general population. All four investigated forms of healthcare utilisation showed a pattern of decreasing ORs, with ORs below 1 in the oldest age groups. This pattern is consistent with a recently published study from Norway [41] that showed people with ID were more frequently hospitalized at a younger age and less frequently at old age. Low levels of unplanned care might occur if, when reaching older age, people with ID were well monitored by the healthcare system and therefore had their healthcare needs met elsewhere, in particular in primary care settings. Not all parts of the health system were included in the present study and thus, this could not be controlled for. 
Table 2 Mean number of planned somatic inpatient registrations and length of stay among those with at least one registration

\begin{tabular}{|c|c|c|c|c|c|c|c|c|c|c|c|c|c|}
\hline \multirow[b]{2}{*}{ Year } & & \multicolumn{6}{|c|}{ Planned inpatient registrations } & \multicolumn{6}{|l|}{ Planned LOS } \\
\hline & & Age 45-54 & Age 55-64 & Age 65-74 & Age $75-84$ & Age $85-94$ & Trend $p$-value & Age 45-54 & Age 55-64 & Age 65-74 & Age $75-84$ & Age 85-94 & Trend $p$-value \\
\hline \multirow[t]{3}{*}{2002} & ID (SD) & $1.08(0.31)$ & $1.28(0.56)$ & $1.07(0.26)$ & & & 0.087 & $7.36(19.71)$ & 7.40 (8.61) & $3.67(4.12)$ & & & 0.170 \\
\hline & G (SD) & $1.19(0.39)$ & $1.25(0.51)$ & $1.20(0.41)$ & & & 0.812 & $6.24(7.26)$ & $6.53(14.32)$ & $5.04(4.26)$ & & & 0.440 \\
\hline & $p$-value ${ }^{a}$ & 0.035 & 0.882 & 0.259 & & & & 0.040 & 0.214 & 0.184 & & & \\
\hline \multirow[t]{3}{*}{2003} & ID (SD) & $1.16(0.44)$ & $1.19(0.43)$ & $1.21(0.72)$ & & & 0.627 & $8.56(14.55)$ & $5.29(6.31)$ & $6.83(10.75)$ & & & 0.737 \\
\hline & G (SD) & $1.37(1.32)$ & $1.18(0.52)$ & $1.07(0.37)$ & & & 0.106 & $6.48(15.02)$ & $7.23(20.10)$ & $7.70(13.64)$ & & & 0.151 \\
\hline & $p$-value ${ }^{a}$ & 0.640 & 0.642 & 0.417 & & & & 0.369 & 0.148 & 0.434 & & & \\
\hline \multirow[t]{3}{*}{2004} & ID (SD) & $1.24(0.56)$ & $1.10(0.31)$ & $1.22(0.64)$ & $1.00(0.00)$ & & 0.178 & $8.90(17.83)$ & $5.01(6.07)$ & 7.89 (9.02) & $8.20(7.16)$ & & 0.282 \\
\hline & G (SD) & $1.19(0.53)$ & $1.21(0.59)$ & $1.27(0.65)$ & $1.67(1.63)$ & & 0.544 & $4.49(5.66)$ & $6.08(9.56)$ & $7.05(8.81)$ & 8.00 (9.19) & & 0.023 \\
\hline & $p$-value ${ }^{a}$ & 0.448 & 0.409 & 0.590 & 0.361 & & & 0.126 & 0.648 & 0.422 & 1.000 & & \\
\hline \multirow[t]{3}{*}{2005} & ID (SD) & $1.25(0.53)$ & $1.15(0.47)$ & $1.21(0.41)$ & $1.00(0.00)$ & & 0.280 & $4.37(5.29)$ & $5.67(5.96)$ & $9.17(11.15)$ & $5.14(5.18)$ & & 0.024 \\
\hline & $\mathrm{G}(\mathrm{SD})$ & $1.15(0.47)$ & $1.19(0.61)$ & $1.11(0.39)$ & $1.10(0.32)$ & & 0.786 & $4.49(4.29)$ & $5.87(15.65)$ & $5.02(4.94)$ & $4.30(2.58)$ & & 0.800 \\
\hline & $p$-value ${ }^{a}$ & 0.168 & 0.955 & 0.179 & 0.403 & & & 0.194 & 0.127 & 0.257 & 0.921 & & \\
\hline \multirow[t]{3}{*}{2006} & ID (SD) & $1.23(0.71)$ & $1.26(0.61)$ & $1.51(0.95)$ & $1.00(0.00)$ & & 0.267 & $6.07(10.41)$ & $8.37(21.82)$ & $7.17(11.23)$ & $3.30(2.98)$ & & 0.255 \\
\hline & G (SD) & $1.33(0.72)$ & $1.23(0.65)$ & $1.24(0.48)$ & $1.81(1.80)$ & & 0.484 & $5.50(6.23)$ & 4.65 (7.53) & $5.04(6.55)$ & $23.06(56.96)$ & & 0.318 \\
\hline & $p$-value ${ }^{a}$ & 0.336 & 0.485 & 0.243 & 0.055 & & & 0.695 & 0.500 & 0.601 & 0.134 & & \\
\hline \multirow[t]{3}{*}{2007} & ID (SD) & $1.30(0.85)$ & $1.20(0.49)$ & $1.29(0.79)$ & $1.20(0.63)$ & & 0.991 & 8.98 (19.92) & $5.94(8.00)$ & $6.10(5.55)$ & $5.80(7.21)$ & & 0.056 \\
\hline & G (SD) & $1.32(0.94)$ & $1.34(0.80)$ & $1.35(0.68)$ & $1.25(0.72)$ & & 0.545 & $11.62(37.18)$ & 7.46 (10.68) & $8.94(11.64)$ & 7.05 (17.63) & & 0.547 \\
\hline & $p$-value ${ }^{a}$ & 0.945 & 0.432 & 0.364 & 0.738 & & & 0.673 & 0.298 & 0.667 & 0.859 & & \\
\hline \multirow[t]{3}{*}{2008} & ID (SD) & $1.13(0.40)$ & $1.28(0.67)$ & $1.11(0.32)$ & $1.14(0.38)$ & & 0.998 & $4.91(6.94)$ & $8.84(15.49)$ & $6.09(8.62)$ & $6.14(6.64)$ & & 0.772 \\
\hline & G (SD) & $1.17(0.47)$ & $1.28(0.71)$ & $1.22(0.52)$ & $1.42(0.51)$ & & 0.463 & $6.55(10.53)$ & $5.23(7.58)$ & $4.37(4.54)$ & $7.50(7.93)$ & & 0.440 \\
\hline & $p$-value ${ }^{a}$ & 0.675 & 1.000 & 0.358 & 0.228 & & & 0.926 & 0.043 & 0.789 & 0.608 & & \\
\hline \multirow[t]{3}{*}{2009} & $\mathrm{ID}(\mathrm{SD})$ & $1.20(0.40)$ & $1.22(0.62)$ & $1.24(0.62)$ & $1.19(0.54)$ & & 0.513 & $8.33(14.65)$ & $7.22(13.69)$ & $7.80(9.50)$ & $8.06(14.34)$ & & 0.737 \\
\hline & G (SD) & $1.21(0.79)$ & $1.25(0.56)$ & $1.21(0.63)$ & $1.39(0.88)$ & & 0.680 & $7.11(17.56)$ & $5.51(7.05)$ & $6.28(7.65)$ & $9.32(12.56)$ & & 0.037 \\
\hline & $p$-value ${ }^{a}$ & 0.341 & 0.604 & 0.710 & 0.349 & & & 0.286 & 0.908 & 0.837 & 0.369 & & \\
\hline \multirow[t]{3}{*}{2010} & ID (SD) & $1.17(0.51)$ & $1.13(0.39)$ & $1.19(0.54)$ & $1.33(0.50)$ & & 0.322 & $6.17(12.70)$ & $6.47(10.10)$ & $6.36(8.18)$ & $22.89(20.82)$ & & 0.008 \\
\hline & G (SD) & $1.41(0.73)$ & $1.22(0.52)$ & $1.28(0.70)$ & $1.26(0.45)$ & & 0.983 & $11.91(21.98)$ & $8.40(22.56)$ & $5.23(6.13)$ & $6.82(7.43)$ & & 0.458 \\
\hline & $p$-value ${ }^{a}$ & 0.212 & 0.171 & 0.285 & 0.675 & & & 0.056 & 0.709 & 0.328 & 0.009 & & \\
\hline \multirow[t]{3}{*}{2011} & $\mathrm{ID}(\mathrm{SD})$ & $1.08(0.29)$ & $1.15(0.54)$ & $1.27(0.64)$ & $1.17(0.46)$ & & 0.111 & $3.00(2.70)$ & $5.62(7.58)$ & $7.17(11.82)$ & $6.87(7.40)$ & & 0.211 \\
\hline & $\mathrm{G}(\mathrm{SD})$ & $1.25(0.46)$ & $1.30(0.72)$ & $1.30(0.63)$ & $1.39(0.97)$ & & 0.368 & $6.25(5.12)$ & $4.51(4.97)$ & $5.64(6.83)$ & $10.55(11.48)$ & & 0.023 \\
\hline & $p$-value ${ }^{a}$ & 0.319 & 0.019 & 0.581 & 0.367 & & & 0.140 & 0.784 & 0.686 & 0.193 & & \\
\hline \multirow[t]{3}{*}{2012} & ID (SD) & & $1.21(0.56)$ & $1.23(0.56)$ & $1.15(0.37)$ & & 0.767 & & $6.83(13.83)$ & $5.68(7.60)$ & $4.50(4.94)$ & & 0.716 \\
\hline & G (SD) & & $1.30(0.69)$ & $1.27(0.59)$ & $1.37(1.00)$ & & 0.789 & & $6.40(14.15)$ & $5.63(5.60)$ & $7.90(10.35)$ & & 0.062 \\
\hline & $p$-value ${ }^{a}$ & & 0.279 & 0.442 & 0.483 & & & & 0.905 & 0.075 & 0.358 & & \\
\hline
\end{tabular}


Table 3 Mean number of unplanned somatic inpatient registration and length of stay among those with at least one registration

\begin{tabular}{|c|c|c|c|c|c|c|c|c|c|c|c|c|c|}
\hline \multirow[b]{2}{*}{ Year } & & \multicolumn{6}{|c|}{ Unplanned inpatient registrations } & \multicolumn{6}{|c|}{ Unplanned LOS } \\
\hline & & Age 45-54 & Age 55-64 & Age $65-74$ & Age $75-84$ & Age 85-94 & Trend $p$-value ${ }^{\mathrm{b}}$ & Age 45-54 & Age $55-64$ & Age 65-74 & Age $75-84$ & Age 85-94 & Trend $p$-value ${ }^{\mathrm{b}}$ \\
\hline \multirow[t]{3}{*}{2002} & ID (SD) & $1.59(1.65)$ & $1.54(1.28)$ & $1.15(0.41)$ & $1.44(0.73)$ & & 0.281 & $5.94(7.28)$ & $8.14(12.37)$ & $6.31(7.69)$ & $9.67(9.21)$ & & 0.116 \\
\hline & $\mathrm{G}(\mathrm{SD})$ & $1.39(1.28)$ & $1.37(0.94)$ & $1.27(0.59)$ & $1.38(0.74)$ & & 0.528 & $4.19(7.14)$ & $3.60(4.29)$ & $5.86(9.68)$ & $5.63(7.63)$ & & 0.035 \\
\hline & $p$-value ${ }^{a}$ & 0.028 & 0.178 & 0.281 & 0.764 & & & $<0.001$ & $<0.001$ & 0.482 & 0.209 & & \\
\hline \multirow[t]{3}{*}{2003} & ID (SD) & $1.47(1.25)$ & $1.45(1.04)$ & $1.62(1.30)$ & $1.36(0.92)$ & & 0.262 & $6.08(9.27)$ & $7.20(11.05)$ & $8.21(11.16)$ & $3.18(1.47)$ & & 0.001 \\
\hline & G (SD) & $1.33(0.92)$ & $1.38(0.93)$ & $1.29(0.64)$ & $1.08(0.28)$ & & 0.812 & $4.09(4.58)$ & $4.83(6.41)$ & $4.78(6.52)$ & $3.38(2.90)$ & & 0.972 \\
\hline & $p$-value ${ }^{a}$ & 0.435 & 0.360 & 0.259 & 0.420 & & & 0.229 & 0.001 & 0.005 & 0.930 & & \\
\hline \multirow[t]{3}{*}{2004} & ID (SD) & $1.66(1.44)$ & $1.46(1.11)$ & $1.41(0.84)$ & $1.19(0.40)$ & & 0.053 & $7.44(10.18)$ & 7.44 (11.24) & $9.41(15.55)$ & $8.00(9.35)$ & & 0.200 \\
\hline & G (SD) & $1.53(1.46)$ & $1.27(0.71)$ & $1.84(1.12)$ & $1.14(0.47)$ & & 0.470 & $5.33(11.25)$ & $3.72(4.53)$ & 7.31 (10.03) & $5.00(4.59)$ & & 0.038 \\
\hline & $p$-value ${ }^{a}$ & 0.287 & 0.179 & 0.004 & 0.435 & & & $<0.001$ & $<0.001$ & 0.356 & 0.341 & & \\
\hline \multirow[t]{3}{*}{2005} & ID (SD) & $1.57(1.57)$ & $1.67(1.58)$ & $1.38(0.84)$ & $1.55(1.10)$ & & 0.950 & $6.96(14.94)$ & $7.72(12.11)$ & 7.34 (7.41) & $6.55(7.66)$ & & 0.007 \\
\hline & G (SD) & $1.37(0.78)$ & $1.32(0.70)$ & 1.78 (1.65) & $1.43(0.79)$ & & 0.459 & $4.73(6.55)$ & $4.24(6.82)$ & $5.48(7.73)$ & $6.75(8.16)$ & & 0.406 \\
\hline & $p$-value ${ }^{a}$ & 0.664 & 0.073 & 0.271 & 0.768 & & & 0.156 & $<0.001$ & 0.014 & 0.627 & & \\
\hline \multirow[t]{3}{*}{2006} & ID (SD) & $1.38(0.91)$ & $1.53(1.46)$ & $1.39(1.00)$ & $1.26(0.53)$ & & 0.794 & $5.80(8.94)$ & $5.92(8.67)$ & $6.87(8.35)$ & $6.70(5.86)$ & & 0.002 \\
\hline & G (SD) & $1.36(0.83)$ & $1.46(1.10)$ & $1.37(1.07)$ & $1.26(0.68)$ & & 0.685 & $6.23(13.23)$ & $5.15(10.18)$ & $5.17(8.09)$ & $7.16(9.53)$ & & 0.390 \\
\hline & $p$-value ${ }^{a}$ & 0.886 & 0.585 & 0.898 & 0.623 & & & 0.485 & 0.023 & 0.005 & 0.319 & & \\
\hline \multirow[t]{3}{*}{2007} & ID (SD) & $1.48(0.99)$ & $1.63(1.50)$ & $1.41(0.91)$ & $1.30(0.53)$ & & 0.414 & $7.31(11.77)$ & $8.33(10.24)$ & 7.95 (9.79) & $6.00(4.22)$ & & 0.045 \\
\hline & G (SD) & $1.44(1.12)$ & $1.51(1.51)$ & $1.42(0.82)$ & $1.72(0.92)$ & & 0.010 & $4.27(8.44)$ & $5.54(12.50)$ & $6.35(7.80)$ & $6.62(6.26)$ & & $<0.001$ \\
\hline & $p$-value & 0.315 & 0.084 & 0.579 & 0.029 & & & 0.001 & $<0.001$ & 0.095 & 0.816 & & \\
\hline \multirow[t]{3}{*}{2008} & ID (SD) & $1.47(1.02)$ & $1.65(1.43)$ & $1.37(0.82)$ & $1.45(0.80)$ & & 0.651 & $6.63(11.33)$ & $8.10(10.88)$ & $6.55(6.09)$ & $6.11(5.13)$ & & 0.041 \\
\hline & G (SD) & $1.51(1.27)$ & $1.45(0.93)$ & $1.54(1.34)$ & $1.41(0.89)$ & & 0.736 & $6.02(9.74)$ & $5.78(8.63)$ & $6.64(10.76)$ & $6.67(8.25)$ & & 0.280 \\
\hline & $p$-value ${ }^{a}$ & 0.712 & 0.171 & 0.902 & 0.365 & & & 0.212 & $<0.001$ & 0.003 & 0.311 & & \\
\hline \multirow[t]{3}{*}{2009} & ID (SD) & $1.70(1.77)$ & $1.61(1.19)$ & $1.71(1.27)$ & $1.64(1.40)$ & & 0.672 & $8.55(17.95)$ & $8.33(12.55)$ & 8.99 (11.65) & $8.59(8.95)$ & & 0.093 \\
\hline & G (SD) & $1.64(1.39)$ & $1.38(0.86)$ & 1.65 (1.16) & $1.68(1.33)$ & & 0.053 & $5.09(7.98)$ & $4.66(6.86)$ & 7.24 (8.77) & $8.29(8.44)$ & & $<0.001$ \\
\hline & $p$-value ${ }^{a}$ & 0.873 & 0.011 & 0.989 & 0.560 & & & 0.003 & $<0.001$ & 0.267 & 0.764 & & \\
\hline \multirow[t]{3}{*}{2010} & ID (SD) & $1.68(1.65)$ & $1.63(1.20)$ & $1.55(1.26)$ & $1.59(0.93)$ & $1.58(0.67)$ & 0.698 & 7.08 (10.96) & $7.15(9.81)$ & 7.83 (9.72) & $7.90(9.16)$ & $8.75(7.71)$ & 0.009 \\
\hline & G (SD) & $1.48(1.04)$ & $1.44(1.00)$ & 1.57 (1.19) & $1.55(1.09)$ & $1.33(0.90)$ & 0.279 & $7.58(14.44)$ & $4.67(6.40)$ & $7.12(10.89)$ & $8.75(9.95)$ & $8.87(7.82)$ & $<0.001$ \\
\hline & $p$-value ${ }^{a}$ & 0.634 & 0.027 & 0.526 & 0.374 & 0.095 & & 0.120 & $<0.001$ & 0.045 & 0.868 & 1.000 & \\
\hline \multirow[t]{3}{*}{2011} & ID (SD) & $1.56(1.41)$ & $1.71(1.23)$ & $1.62(1.33)$ & $1.75(1.40)$ & $1.82(1.07)$ & 0.996 & $5.36(7.72)$ & $8.19(10.89)$ & $8.03(9.89)$ & $8.58(9.89)$ & 6.47 (5.79) & 0.016 \\
\hline & G (SD) & $1.09(0.29)$ & $1.46(1.12)$ & $1.65(1.47)$ & $1.79(1.30)$ & $1.73(1.22)$ & $<0.001$ & $3.55(5.46)$ & $5.41(12.11)$ & 7.69 (11.68) & $11.64(14.76)$ & $7.47(4.64)$ & $<0.001$ \\
\hline & $p$-value ${ }^{a}$ & 0.076 & $<0.001$ & 0.729 & 0.737 & 0.674 & & 0.203 & $<0.001$ & 0.090 & 0.207 & 0.471 & \\
\hline \multirow[t]{3}{*}{2012} & ID (SD) & & $1.70(1.37)$ & 1.79 (1.40) & $1.66(1.09)$ & $1.71(1.12)$ & 0.276 & & $7.83(11.50)$ & $8.57(10.07)$ & 11.11 (14.91) & $8.13(6.90)$ & $<0.001$ \\
\hline & $\mathrm{G}(\mathrm{SD})$ & & $1.64(1.74)$ & $1.69(1.27)$ & $1.71(1.32)$ & $2.04(1.35)$ & 0.021 & & $6.19(10.06)$ & $8.07(11.05)$ & $9.82(11.54)$ & $16.75(17.62)$ & $<0.001$ \\
\hline & $p$-value ${ }^{a}$ & & 0.049 & 0.137 & 0.886 & 0.299 & & & 0.005 & 0.006 & 0.299 & 0.061 & \\
\hline
\end{tabular}


Table 4 Mean number of physician visits in somatic outpatient specialist care among those with at least one visit

\begin{tabular}{|c|c|c|c|c|c|c|c|c|c|c|c|c|c|}
\hline \multirow[b]{2}{*}{ Year } & & \multicolumn{6}{|c|}{ Planned outpatient visits to physician } & \multicolumn{6}{|c|}{ Unplanned outpatient visits to physician } \\
\hline & & Age 45-54 & Age 55-64 & Age $65-74$ & Age 75-84 & Age 85-94 & Trend $p$-value & Age 45-54 & Age 55-64 & Age 65-74 & Age $75-84$ & Age 85-94 & Trend $p$-value \\
\hline \multirow[t]{3}{*}{2002} & ID (SD) & $1.59(1.11)$ & $1.74(1.95)$ & $1.98(2.10)$ & $1.13(0.35)$ & & 0.825 & $1.71(1.83)$ & $1.79(2.94)$ & $1.63(2.47)$ & & & 0.404 \\
\hline & $\mathrm{G}(\mathrm{SD})$ & $1.85(1.47)$ & $1.70(1.23)$ & $1.82(1.25)$ & $1.83(1.00)$ & & 0.529 & $1.19(0.49)$ & $1.41(0.96)$ & $1.23(0.55)$ & & & 0.069 \\
\hline & $p$-value ${ }^{a}$ & 0.017 & 0.101 & 0.574 & 0.057 & & & $<0.001$ & 0.309 & 0.675 & & & \\
\hline \multirow[t]{3}{*}{2003} & ID (SD) & $1.62(1.44)$ & $1.72(2.05)$ & 1.68 (1.19) & $1.41(0.80)$ & & 0.318 & $1.78(2.24)$ & $1.72(2.64)$ & $1.24(0.43)$ & $1.20(0.45)$ & & 0.945 \\
\hline & $\mathrm{G}(\mathrm{SD})$ & $1.72(1.72)$ & $1.78(1.40)$ & $1.73(1.34)$ & $2.00(1.43)$ & & 0.290 & $1.25(0.64)$ & $1.22(0.73)$ & $1.32(0.64)$ & $1.14(0.38)$ & & 0.946 \\
\hline & $p$-value ${ }^{a}$ & 0.093 & 0.076 & 0.984 & 0.147 & & & 0.014 & 0.001 & 0.956 & 0.802 & & \\
\hline \multirow[t]{3}{*}{2004} & ID (SD) & $1.79(1.85)$ & $1.73(1.54)$ & $1.61(1.00)$ & $1.50(0.99)$ & & 0.738 & $1.55(1.32)$ & $1.70(3.41)$ & $1.37(0.82)$ & $1.38(0.74)$ & & 0.625 \\
\hline & G (SD) & 1.80 (1.96) & $1.89(1.96)$ & $1.86(1.42)$ & $2.06(1.76)$ & & 0.022 & $1.25(0.58)$ & $1.22(0.70)$ & $1.42(0.91)$ & $1.45(0.52)$ & & 0.315 \\
\hline & $p$-value ${ }^{a}$ & 0.871 & 0.254 & 0.105 & 0.191 & & & 0.019 & 0.010 & 0.962 & 0.527 & & \\
\hline \multirow[t]{3}{*}{2005} & ID (SD) & $1.68(1.33)$ & $1.81(1.69)$ & $1.71(1.40)$ & $1.72(1.27)$ & & 0.835 & $1.42(1.01)$ & $1.62(1.97)$ & $1.34(0.75)$ & $1.50(0.84)$ & & 0.843 \\
\hline & G (SD) & $1.80(2.14)$ & $1.89(1.71)$ & $1.81(1.38)$ & $1.80(1.09)$ & & 0.080 & $1.26(0.72)$ & $1.34(0.81)$ & $1.18(0.51)$ & $1.11(0.33)$ & & 0.901 \\
\hline & $p$-value ${ }^{a}$ & 0.885 & 0.254 & 0.131 & 0.590 & & & 0.101 & 0.586 & 0.219 & 0.273 & & \\
\hline \multirow[t]{3}{*}{2006} & ID (SD) & $1.77(1.50)$ & $1.95(2.56)$ & 1.70 (1.47) & $1.78(1.40)$ & & 0.984 & $1.40(0.86)$ & $1.54(1.67)$ & $1.42(0.85)$ & $1.50(0.84)$ & & 0.948 \\
\hline & G (SD) & $1.78(1.88)$ & $2.16(3.21)$ & $2.16(2.47)$ & $2.00(1.37)$ & & 0.006 & $1.21(0.69)$ & $1.28(0.65)$ & $1.35(0.97)$ & $1.32(0.58)$ & & 0.070 \\
\hline & $p$-value ${ }^{a}$ & 0.441 & 0.927 & 0.049 & 0.195 & & & 0.009 & 0.590 & 0.473 & 0.657 & & \\
\hline \multirow[t]{3}{*}{2007} & ID (SD) & $1.64(1.32)$ & $1.76(1.95)$ & $1.63(1.08)$ & $1.50(0.77)$ & & 0.342 & $1.45(1.55)$ & $1.49(1.38)$ & $1.32(0.93)$ & $1.28(0.46)$ & & 0.297 \\
\hline & G (SD) & $1.85(1.57)$ & $1.86(1.87)$ & $2.19(2.80)$ & $2.59(4.07)$ & & 0.134 & $1.26(0.73)$ & $1.30(0.85)$ & $1.21(0.49)$ & $1.33(0.55)$ & & 0.353 \\
\hline & $p$-value ${ }^{a}$ & 0.032 & 0.104 & 0.050 & 0.092 & & & 0.053 & 0.076 & 0.967 & 0.819 & & \\
\hline \multirow[t]{3}{*}{2008} & ID (SD) & $1.49(0.97)$ & $1.83(2.03)$ & $1.52(0.90)$ & $1.75(1.18)$ & & 0.203 & $1.42(1.01)$ & $1.51(1.50)$ & $1.34(0.72)$ & $1.25(0.51)$ & & 0.746 \\
\hline & G (SD) & $2.36(3.85)$ & $1.89(2.37)$ & $2.00(2.22)$ & $2.25(3.32)$ & & 0.415 & $1.30(0.70)$ & $1.31(0.90)$ & $1.35(0.74)$ & $1.28(0.60)$ & & 0.722 \\
\hline & $p$-value ${ }^{a}$ & $<0.001$ & 0.571 & 0.008 & 0.318 & & & 0.815 & 0.132 & 0.877 & 0.930 & & \\
\hline \multirow[t]{3}{*}{2009} & ID (SD) & $1.51(0.87)$ & $1.67(1.15)$ & $1.71(1.73)$ & $1.72(1.09)$ & & 0.453 & 1.59 (1.74) & $1.31(0.81)$ & $1.47(1.11)$ & $1.36(0.59)$ & & 0.649 \\
\hline & G (SD) & $1.87(1.40)$ & $2.03(2.32)$ & $1.97(1.57)$ & $2.23(1.94)$ & & 0.024 & $1.27(0.78)$ & $1.47(2.10)$ & $1.45(0.78)$ & 1.45 (1.15) & & 0.034 \\
\hline & $p$-value ${ }^{a}$ & 0.047 & 0.027 & 0.001 & 0.062 & & & 0.080 & 0.238 & 0.495 & 0.487 & & \\
\hline \multirow[t]{3}{*}{2010} & ID (SD) & $1.57(1.06)$ & $1.72(1.25)$ & $1.88(2.06)$ & $1.59(0.89)$ & $1.80(1.03)$ & 0.088 & $1.45(1.45)$ & $1.46(1.19)$ & $1.27(0.60)$ & $1.32(0.73)$ & $1.20(0.45)$ & 0.246 \\
\hline & $\mathrm{G}(\mathrm{SD})$ & 2.21 (3.38) & $2.20(3.14)$ & $2.11(2.15)$ & $2.41(4.08)$ & $1.71(1.31)$ & 0.107 & $1.24(0.60)$ & $1.28(0.67)$ & $1.32(0.80)$ & $1.26(0.68)$ & $1.00(0.00)$ & 0.962 \\
\hline & $p$-value ${ }^{a}$ & 0.116 & 0.006 & 0.050 & 0.035 & 0.449 & & 0.173 & 0.283 & 0.938 & 0.753 & 0.273 & \\
\hline \multirow[t]{3}{*}{2011} & ID (SD) & $1.79(1.12)$ & 1.85 (1.69) & $1.89(2.13)$ & $1.73(1.20)$ & $1.71(1.11)$ & 0.766 & $1.36(0.98)$ & $1.45(1.03)$ & $1.29(0.76)$ & $1.45(0.70)$ & $1.00(0.00)$ & 0.586 \\
\hline & $\mathrm{G}(\mathrm{SD})$ & $1.76(1.50)$ & $2.25(3.41)$ & $2.33(2.82)$ & $2.13(1.74)$ & $1.86(0.95)$ & 0.002 & $1.31(0.52)$ & $1.28(0.82)$ & $1.44(0.94)$ & $1.29(0.87)$ & $1.33(0.71)$ & 0.564 \\
\hline & $p$-value ${ }^{a}$ & 0.172 & 0.049 & 0.001 & 0.063 & 0.544 & & 0.489 & 0.006 & 0.136 & 0.025 & 0.198 & \\
\hline \multirow[t]{3}{*}{2012} & ID (SD) & & $1.90(2.40)$ & $1.83(1.58)$ & $1.59(1.16)$ & $1.63(0.81)$ & 0.233 & & $1.52(1.42)$ & $1.45(1.04)$ & $1.20(0.46)$ & $1.55(0.82)$ & 0.821 \\
\hline & $\mathrm{G}(\mathrm{SD})$ & & $2.01(1.78)$ & $2.55(3.20)$ & $2.44(2.11)$ & $1.72(1.09)$ & $<0.001$ & & $1.32(0.74)$ & $1.29(0.71)$ & $1.27(0.57)$ & $1.50(0.89)$ & 0.695 \\
\hline & $p$-value ${ }^{a}$ & & 0.005 & $<0.001$ & $<0.001$ & 0.859 & & & 0.389 & 0.043 & 0.574 & 0.790 & \\
\hline
\end{tabular}

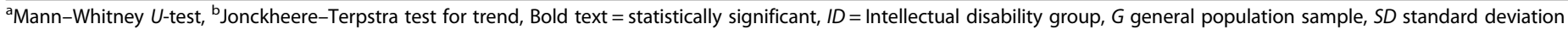


Nevertheless, if people with ID were sufficiently monitored, this should reasonably lead to high levels of planned healthcare, especially considering their reduced health in comparison with the general population, as shown in a previous study using the same sample as in the present study [3]. However, this is contradicted by our finding that people with ID in the oldest age groups had lower proportions of planned in- and outpatient care than the general population. People with high utilisation in one provider have been demonstrated to also have high utilisation in other areas [51]. It has been reported that people with ID with more than 24 outpatient visits had 2.81 greater odds of being hospitalized [33], meaning it is unlikely that those with low healthcare utilisation in the present study would have high utilisation of other services (e.g., primary care). Regardless of whether or not health needs are met elsewhere in the health system, it is reasonable to expect that older people with ID, who are known to have more diseases, will have higher rates of healthcare use, or at least rates that are not lower than in the general population.

In addition, the results of the present study showed that for those with at least one planned visit to physician in outpatient care, the ID group had fewer visits compared with the control group (Table 4). The reason why people with ID have fewer registrations of planned healthcare needs further study. Some studies have suggested that people with ID may have difficulty accessing the health system $[14,52]$, with lower rates of preventive healthcare such as breast and cervical cancer screening $[4,10]$ and influenza vaccination $[10,53]$. The health system's different treatment of people with ID compared with the general population is supported by several reviews [54-57]. These reviews identified experiences of people with ID, parents, carers and/or healthcare staff of barriers that prevent access to quality healthcare. Reported barriers include experiencing the healthcare/hospital as a fearful encounter, negative attitudes and discrimination, lack of knowledge and formal training among staff, communication difficulties, and the diagnostic uncertainty that may be present when the person with ID has problems in verbal expressions of pain, anxiety, and distress [54-57].

Another possible explanation for our results is that in people with ID, the oldest age groups are healthier than their younger peers. This is somewhat contradicted by the in-group comparison of the ID group (Fig. 3) that revealed an increase of unplanned healthcare utilisation with age. Although it may seem unlikely that people with ID should become healthier with increasing age, this may occur if there was a selection of healthier people in the oldest age groups; that is, if those with severe and profound ID were less likely to survive into older age. This may result in higher proportions of people with mild or moderate ID in the oldest age groups compared with the younger age groups. However, even if there is such an overrepresentation these would be expected to suffer from age-related diagnoses and thus, rates of healthcare utilisation, to the same extent as their peers in the general population. Nonetheless, in the present study, we had no information about the severity of ID, which might have resulted in lower healthcare utilisation in the older age groups, and the results should therefore be interpreted with some caution.

Some limitations are mentioned above (lack of primary care data, the risk of a cohort effect, and not being able to discriminate levels of ID severity). In addition, only two variables was used when matching the control group (year of birth and sex) which might have increased the risk of selection bias and reduced internal validity. For example, the sample was not matched on place of residence, meaning that the ID and control group participants might have lived in different parts of the country with different distances to healthcare facilities, which might in turn have affected access to and utilisation of healthcare. As no information about place of residence for the total sample was available we do not know whether this caused an under- or overestimation in the general population sample. Socioeconomic variables were not considered as matching variables. There are studies reporting that people with ID have lower socioeconomic status (SES) than the general population [42, 58 ] and that poor SES is associated with lower access of healthcare in many countries $[31,59]$, including Sweden $[45,60,61]$. However, SES may be a link in the casual chain between ID and utilisation of healthcare and thus, controlling for these variables would also remove potential effects of the ID itself. There are also studies that have been unable to find associations between SES and healthcare utilisation [30,32]. People with ID are predisposed to several conditions, such as lower SES, and the aim of this study was not to explore explanatory factors such as a certain condition or diagnosis, but to explore how the group of people with ID utilised healthcare utilisation in relation to the general population. In addition, the healthcare system in Sweden is mainly funded by taxes, i.e., all people regardless of income level are supposed to have equal access to healthcare. An increased number of matching variables would also make it more difficult to make an unbiased random selection of the general population sample. The results in this study are due to ID, to variables closely related to ID, or to variables that are consequences of ID and interpretations should be made with this in mind.

A person may have several registrations during one hospital stay (defined as one date for admission and one date for discharge), where some registrations could be planned and others unplanned. When the registrations were merged into hospital stay it was not possible to 
determine whether the stay was planned or unplanned. This means that the numbers in Table 3 are higher than the actual number of hospital stays. When comparing the total number of registrations and number of hospital stays, the number of registrations was about $5 \%$ higher than hospital stays. Comparisons with other studies using hospital stay as an outcome should be made with this in mind.

In this study, the results of 398 statistical analyses are presented. Such a high number of tests may be a threat to statistical validity, as it increases the risk of mass significance (i.e., type I error) [62] with approximately a $5 \%$ likelihood (26 tests) of incorrectly rejecting a null hypothesis. To reduce the risk of type I errors, conclusions are drawn on the patterns of the results rather than single $p$-values.

A strength of this study was that the registrations in the NPR are connected to the reimbursement system in Sweden, and the coverage is therefore generally high. For inpatient care, the coverage is almost $100 \%$, but coverage of outpatient care is lower, about $80 \%$ [63]. The main reason for this lower number is a greater proportion of private providers for outpatient care compared with inpatient care, and more data from these are missing than data from public providers (coverage almost $100 \%$ ) [63]. Ludvigsson and colleagues [64] have investigated the validity of inpatient data in the Swedish NPR. They performed a review of 132 papers [64] with focus on registrations of diagnoses. The positive predictive value differed between diagnoses, but was in general between 85-95\%, and they concluded that the validity was high for most diagnoses [64].

The ID group in the present study were identified through a public national register and comprised a large group of people who were registered with ID during 2012 and who, at that time, were aged 55 years or older. A matched control group was also included, with both groups followed retrospectively for 11 years and included a very large set of data from the NPR. To the best of our knowledge, there is no other study investigating older people with ID covering such a large sample and over such a long time period. Altogether, a sample covering all people who received support and social services under the Act concerning Support and Service for Persons with Certain Functional Impairments [47], the matched general population sample, and the high validity of the public mandatory NPR, increases the external validity, meaning that these results may be generalized to similar contexts.

\section{Conclusions}

The ID group had, in the youngest age groups, higher proportions of planned and unplanned in- and outpatient care than the control group. In the oldest age groups, the ID group had lower or similar proportions of unplanned care and lower proportions of planned care than the general population. It is likely that people with ID have reduced access to healthcare, because of their predisposition to having a low SES, which in turn is related to lower access. More research is needed to determine underlying reasons for this unique healthcare utilisation pattern.

\section{Additional file}

Additional file 1: Data concerning differences in proportions with at least one visit registration with respect to four different outcomes: planned/ unplanned inpatient registrations/outpatient physician visits(year, and age group, odds ratios (OR) with $95 \%$ confidence intervals) and data concerning differences in number of visits, registrations and LOS for individuals with at least one registration/visit (mean, standarddeviations, $p$-values for MannWhitney U-tests, and Jonckheere-Terpstra tests for trend). (XLSX 53 kb)

\section{Abbreviations}

ID: Intellectual disabilities; LOS: Length of stay; NPR: National Patient Register; OR: Odds ratio; SES: Socioeconomic status

\section{Acknowledgements}

We would like to acknowledge the cooperation of the FUB, The Swedish National Association for Persons with Intellectual Disability.

\section{Funding}

This study was funded by FORTE — the Swedish Research Council for Health, Working Life and Welfare no 2014-4753, and the Faculty of Medicine, Lund University, Sweden. The funding bodies had no role in the design of the study, collection, analysis, interpretation of data, or writing the manuscript.

\section{Availability of data and materials}

According to the data policy, data need to be publicly available to allow other researchers to be able to reproduce the study. However, the data in the present study contain sensitive information on a very vulnerable group (i.e., people with intellectual disability). Even though the data are anonymized, they may contain sufficient detail to enable identification of single individuals. Therefore, when approving this study, the Regional Ethical Review Board in Lund made severe restrictions regarding access to the data. This means we are not be able to provide our data to other researchers. However, as our database was compiled from registry data only, other researchers may contact Statistics Sweden and the Swedish National Board of Health and Welfare to get access to the different registries included, and thereby recreate the database. For more information about the restrictions imposed by the ethics committee, or how to access the data at Statistics Sweden and the Swedish National Board of Health and Welfare, please contact the project leader, Professor Gerd Ahlström (gerd.ahlstrom@med.lu.se).Information on how to access registry data is also available on www.scb.se/en_/ (Statistics Sweden) and www.socialstyrelsen.se/ english (National Board of Health and Welfare).

\section{Authors' contributions \\ MS participated in the design of the study and in the collection of data, performed the analysis and interpretation of data, and drafted the manuscript GA participated in the design of the study, and helped in the interpretation of the data and drafting of the manuscript. AA participated in the design of the study, and helped in the interpretation of the data and drafting of the manuscript. JK participated in the design of the study, and helped in the interpretation of the data in drafting the manuscript. All authors read and approved the final manuscript.}

\section{Competing interests}

The authors declare that they have no competing interests.

Consent for publication

Not applicable. 


\section{Ethics approval and consent to participate}

The study is part of a project called OID-Health (Older persons with intellectual disability and healthy aging), which used an anonymized dataset, and did not contain any information that made it possible to link data to any individual. The study was approved by the Regional Ethical Review Board in Lund (diary no 2013/15). The National Board of Health and Welfare and Statistics Sweden performed separate secrecy reviews before providing access to the data. The study was performed in accordance with the Helsinki declaration [65].

\section{Author details}

'Department of Health Sciences, Faculty of Medicine, Lund University, P.O. Box 157, SE-221 00 Lund, Sweden. ${ }^{2}$ Department of Occupational and Environmental Medicine, Lund University, SE-221 85 Lund, Sweden. ${ }^{3}$ Department of Health, Blekinge Institute of Technology, SE-371 45 Karlskrona, Sweden.

Received: 25 March 2016 Accepted: 30 October 2016 Published online: 09 November 2016

\section{References}

1. Fisher K, Kettl P. Aging with mental retardation: increasing population of older adults with MR require health interventions and prevention strategies. Geriatrics. 2005;60(4):26-9.

2. McCarron M, Swinburne J, Burke E, McGlinchey E, Carroll R, McCallion P. Patterns of multimorbidity in an older population of persons with an intellectual disability: results from the intellectual disability supplement to the Irish longitudinal study on aging (IDS-TILDA). Res Dev Disabil. 2013;34(1):521-7.

3. Sandberg M, Ahlström G, Kristensson J. Patterns of Somatic Diagnoses in Older People with Intellectual Disability: A Swedish Eleven Year Case-Control Study of Inpatient Data. J Appl Res Intellect. 2015; doi:10.1111/jar.12230

4. Havercamp SM, Scandlin D, Roth M. Health disparities among adults with developmental disabilities, adults with other disabilities, and adults not reporting disability in North Carolina. Public Health Rep. 2004;119(4):418-26.

5. Draheim CC. Cardiovascular disease prevalence and risk factors of persons with mental retardation. Ment Retard Dev D R. 2006;12(1):3-12.

6. van Schrojenstein Lantman-De Valk HM, Van Den Akker M, Maaskant MA, Haveman MJ, Urlings HF, Kessels AG, Crebolder HF. Prevalence and incidence of health problems in people with intellectual disability. J Intellect Disabil Res. 1997;41(1):42-51.

7. Kapell D, Nightingale B, Rodriguez A, Lee JH, Zigman WB, Schupf N. Prevalence of chronic medical conditions in adults with mental retardation: comparison with the general population. Ment Retard. 1998;36(4):269-79.

8. van Schrojenstein Lantman-De Valk HM, Metsemakers JF, Haveman MJ, Crebolder HF. Health problems in people with intellectual disability in general practice: a comparative study. Fam Pract. 2000;17(5):405-7.

9. de Winter CF, Bastiaanse LP, Hilgenkamp TIM, Evenhuis HM, Echteld MA. Overweight and obesity in older people with intellectual disability. Res Dev Disabil. 2012;33(2):398-405.

10. Havercamp SM, Scott HM. National health surveillance of adults with disabilities, adults with intellectual and developmental disabilities, and adults with no disabilities. Disabil Health J. 2014; doi:10.1016/j.dhjo.2014.11.002

11. Baxter H, Lowe K, Houston H, Jones G, Felce D, Kerr M. Previously unidentified morbidity in patients with intellectual disability. $\mathrm{Br} J \mathrm{Gen}$ Pract. 2006;56(523):93-8.

12. Cooper SA, Smiley E, Morrison J, Williamson A, Allan L. Mental ill-health in adults with intellectual disabilities: prevalence and associated factors. Br J Psychiatry. 2007;190:27-35.

13. Haveman M, Perry J, Salvador-Carulla L, Walsh PN, Kerr M, Van Schrojenstein Lantman-de Valk H, Van Hove G, Berger DM, Azema B, Buono S, et al. Ageing and health status in adults with intellectual disabilities: Results of the European POMONA II study. J Intellect Dev Dis. 2011;36(1):49-60.

14. Hayden MF, Kim SH, DePaepe P. Health status, utilization patterns, and outcomes of persons with intellectual disabilities: review of the literature. Ment Retard. 2005:43(3):175-95.

15. Straetmans JM, van Schrojenstein Lantman-de Valk HM, Schellevis FG, Dinant GJ. Health problems of people with intellectual disabilities: the impact for general practice. Br J Gen Pract. 2007;57(534):64-6.
16. Evenhuis HM, Hermans H, Hilgenkamp TI, Bastiaanse LP, Echteld MA. Frailty and disability in older adults with intellectual disabilities: results from the healthy ageing and intellectual disability study. J Am Geriatr Soc. 2012;60(5):934-8.

17. Janicki MP, Dalton AJ, Henderson CM, Davidson PW. Mortality and morbidity among older adults with intellectual disability: health services considerations. Disabil Rehabil. 1999;21(5/6):284-94.

18. World Health Organization. Ageing and Intellectual Disabilities - Improving Longevity and Promoting Healthy Ageing: Summative Report. Geneva: World Health Organization; 2000.

19. Balogh RS, Hunter D, Ouellette-Kuntz H. Hospital utilization among persons with an intellectual disability, Ontario, Canada, 1995-2001. J Appl Res Intellect. 2005;18(2):181-90

20. Morgan CL, Ahmed Z, Kerr MP. Health care provision for people with a learning disability. Record-linkage study of epidemiology and factors contributing to hospital care uptake. Br J Psychiatry. 2000;176:37-41.

21. Chou YC, Lee YC, Lin LC, Chang AN, Huang WY. Social services utilization by adults with intellectual disabilities and their families. Soc Sci Med. 2008;66(12):2474-85.

22. Hall A, Wood D, Hou T, Zhang J. Patterns in primary health care utilization among individuals with intellectual and developmental disabilities in Florida. Intellect Dev Disab. 2007:45(5):310-22.

23. Halstead SM, Bradley F, Milne S, Wright EC, Hollins SC. Annual primary health care contacts by people with intellectual disabilities: a comparison of three matched groups. J Appl Res Intellect. 2000;13(2):100-7.

24. Hemmings C, O'Hara J, McCarthy J, Holt G, Eoster F, Costello H, Hammond $\mathrm{R}$, Xenitidis K, Bouras N. Comparison of adults with intellectual disabilities and mental health problems admitted to specialist and generic inpatient units. Br J Learn Disabil. 2009;37(2):123-8.

25. Hsu SW, Lin JD, Chiang PH, Chang YC, Tung HJ. Comparison of outpatient services between elderly people with intellectual disabilities and the genera elderly population in Taiwan. Res Dev Disabil. 2012;33(5):1429-36.

26. Janicki MP, Davidson PW, Henderson CM, McCallion P, Taets JD, Force LT, Sulkes SB, Frangenberg E, Ladrigan PM. Health characteristics and health services utilization in older adults with intellectual disability living in community residences. J Intellect Disabil Res. 2002;46(Pt 4):287-98.

27. Levy JM, Botuck S, Rimmerman A. Examining outpatient health care utilization among adults with severe or profound intellectual disabilities living in an urban setting: a brief snap shot. J Soc Work Disabil Rehabil. 2007;6(3):33-45.

28. Lin JD, Loh CH, Yen CF, Li CW, Chwo MJ, Wu JL. Medical care services for people with intellectual disabilities living in the general community: a crosssectional survey of inpatient care utilization in Taiwan, 2001. Disabil Rehabil. 2007;29(18):1411-16.

29. Lin JD, Wu JL, Lee PN. Healthcare needs of people with intellectual disability in institutions in Taiwan: outpatient care utilization and implications. J Intellect Disabil Res. 2003;47(Pt 3):169-80.

30. Lin JD, Wu JL, Lee PN. Utilization of inpatient care and its determinants among persons with intellectual disabilities in day care centres in Taiwan. J Intellect Disabil Res. 2004;48(Pt 7):655-62

31. Lin JD, Yen CF, Loh CH, Hsu SW, Huang HC, Tang CC, Li CW, Wu JL. A crosssectional study of the characteristics and determinants of emergency care utilization among people with intellectual disabilities in Taiwan. Res Dev Disabil. 2006;27(6):657-67.

32. Lin JD, Yen CF, Loh $\mathrm{CH}$, Li CW, Wu JL. Rehabilitation service utilization and determinants among people with an intellectual disability: preliminary findings in Taiwan. Disabil Rehabil. 2006;28(23):1499-506.

33. Loh CH, Lin JD, Choi IC, Yen CF, Hsu SW, Wu JL, Tang CC. Longitudinal analysis of inpatient care utilization among people with intellectual disabilities: 1999-2002. J Intellect Disabil Res. 2007:51(Pt 2):101-8.

34. Long SK, Coughlin TA, Kendall SJ. Access to care among disabled adults on Medicaid. Health Care Financ Rev. 2002:23(4):159-73.

35. Lunsky Y, Lin E, Balogh R, Klein-Geltink J, Bennie J, Wilton AS, Kurdyak P. Are adults with developmental disabilities more likely to visit EDs? Am J Emerg Med. 2011;29(4):463-5.

36. Merrick J, Davidson PW, Morad M, Janicki MP, Wexler O, Henderson CM. Older adults with intellectual disability in residential care centers in Israel: health status and service utilization. Am J Ment Retard. 2004;109(5):413-20.

37. Turk V, Kerry S, Corney R, Rowlands G, Khattran S. Why some adults with intellectual disability consult their general practitioner more than others. J Intellect Disabil Res. 2010;54(9):833-42.

38. Wang D, McDermott $S$, Sease T. Analysis of hospital use for injury among individuals with mental retardation. Inj Control Saf Promot. 2002;9(2):107-11. 
39. Venkat A, Pastin RB, Hegde GG, Shea JM, Cook JT, Culig C. An analysis of ED utilization by adults with intellectual disability. Am J Emerg Med. 2011;29(4):401-11.

40. Wood D, Hall A, Hou T, Wludyka P, Zhang J. Continuity of care to prevent emergency room use among persons with intellectual and developmental disabilities. J Policy Pract Intellect Disabil. 2007:4(4):219-28.

41. Skorpen S, Nicolaisen M, Langballe EM. Hospitalisation in adults with intellectual disabilities compared with the general population in Norway. J Intellect Disabil Res. 2016; doi:10.1111/jir.12255

42. Beange $H$, McElduff A, Baker W. Medical disorders of adults with mental retardation: a population study. Am J Ment Retard. 1995;99(6):595-604

43. Lennartsson C, Heimerson I. Elderly people's health: Health in Sweden: The National Public Health Report 2012. Chapter 5. Scand J Public Health. 2012:40 Suppl 9:95-120.

44. Danielsson M, Talbäck M. Public health: An overview: Health in Sweden: The National Public Health Report 2012. Chapter 1. Scand J Public Health. 2012:40 Suppl 9:6-22

45. Anell A, Glenngard AH, Merkur SM. Sweden: Health system review. Health Syst Transit. 2012;14(5):1-159.

46. SFS 1982:763. Hälso- och sjukvårdslag [The Health and Medical Service Act]. Stockholm: Ministry of Health and Social Affairs; 1982. Swedish

47. SFS 1993:387. Lag om stöd och service till vissa funktionshindrade (LSS) [Act concerning Support and Service for Persons with Certain Functional Impairments)]. Stockholm: Ministry of Health and Social Affairs; 1993. Swedish

48. SFS 2001:453. Socialtjänstlag (The Social Services Act). Stockholm: Ministry of Health and Social Affairs; 2001. Swedish

49. Wilén A, Johannesson I. A new Total population register system: More possibilities and better quality. Örebro; 2002

50. Statistics Sweden: Statistic Sweden startpage. http://www.scb.se/en_/ (2015). Accessed 21 Oct 2015.

51. Condelius A, Edberg AK, Jakobsson U, Hallberg IR. Hospital admissions among people $65+$ related to multimorbidity, municipal and outpatient care. Arch Gerontol Geriatr. 2008;46(1):41-55.

52. Scheepers M, Kerr M, O'hara D, Bainbridge D, Cooper SA, Davis R, Fujiura G, Heller T, Holland A, Krahn G. Reducing health disparity in people with intellectual disabilities: a report from health issues special interest research group of the international association for the scientific study of intellectual disabilities. J Policy Pract Intellect Disabil. 2005;2(3-4):249-55.

53. Lewis MA, Lewis $C E$, Leake $B$, King BH, Lindemann $R$. The quality of health care for adults with developmental disabilities. Public Health Rep. 2002;117(2):174-84.

54. Backer C, Chapman M, Mitchell D. Access to secondary healthcare for people with intellectual disabilities: a review of the literature. J Appl Res Intellect. 2009;22(6):514-25.

55. Bradbury-Jones C, Rattray J, Jones M, Macgillivray S. Promoting the health, safety and welfare of adults with learning disabilities in acute care settings: a structured literature review. J Clin Nurs. 2013;22(11-12):1497-509.

56. lacono T, Bigby C, Unsworth C, Douglas J, Fitzpatrick P. A systematic review of hospital experiences of people with intellectual disability. BMC Health Serv Res. 2014;14:505.

57. Phillips L. Improving care for people with learning disabilities in hospital. Nurs Stand. 2012;26(23):42-8.

58. Emerson E, Hatton C, Robertson J, Baines S. Perceptions of neighbourhood quality, social and civic participation and the self rated health of British adults with intellectual disability: cross sectional study. BMC Public Health. 2014;14:1252.

59. Nie JX, Wang L, Tracy CS, Moineddin R, Upshur RE. A population-based cohort study of ambulatory care service utilization among older adults. J Eval Clin Pract. 2010;16(4):825-31.

60. Socialstyrelsen [The National Board of Health and Welfare]. Tillståndet och utvecklingen inom hälso- och sjukvård samt tandvård - Lägesrapport 2016 [The state and the development of healthcare and dental care - State of play 2016. Stockholm: Socialstyrelsen; 2016. Swedish

61. van Doorslaer E, Masseria C, Koolman X. Inequalities in access to medical care by income in developed countries. CMAJ. 2006;174(2):177-83.

62. Altman DG. Practical Statistics for Medical Research. London: Chapman \& Hall; 1991.

63. Socialstyrelsen [The National Board of Health and Welfare]. Kvalitet och innehåll i patientregistret - Utskrivningar från slutenvården 1964-2007 och besök i specialiserad öppenvård (exklusive primärvårdsbesök) 1997-2007 [Quality and content in the patient register - Discharges from inpatient care 1964-2007 and visits to specialist outpatient care (excl. primary care visits) 1997-2007]. Stockholm: Socialstyrelsen; 2009. Swedish
64. Ludvigsson JF, Andersson E, Ekbom A, Feychting M, Kim JL, Reuterwall C, Heurgren M, Olausson PO. External review and validation of the Swedish national inpatient register. BMC Public Health. 2011;11:450.

65. World Medical Association. World medical association declaration of helsinki: Ethical principles for medical research involving human subjects. JAMA. 2013:310(20):2191-94

\section{Submit your next manuscript to BioMed Central and we will help you at every step:}

- We accept pre-submission inquiries

- Our selector tool helps you to find the most relevant journal

- We provide round the clock customer support

- Convenient online submission

- Thorough peer review

- Inclusion in PubMed and all major indexing services

- Maximum visibility for your research

Submit your manuscript at www.biomedcentral.com/submit

) Biomed Central 